\title{
THE HOT R CORONAE BOREALIS STAR DY CENTAURI IS A BINARY
}

\author{
N. Kameswara RaO ${ }^{1,2}$, David L. Lambert ${ }^{2}$, D. A. García-Hernández ${ }^{3,4}$, C. Simon JefFer ${ }^{5}$, \\ VinCENT M. WOOLF ${ }^{6}$, AND Barbara MCARTHUR ${ }^{2}$ \\ 1 543, 17th Main, IV Sector, HSR Layout, Bangalore 560102, India; nkrao@iiap.res.in \\ 2 The W. J. McDonald Observatory, University of Texas, Austin, TX 78712-1083, USA; dll@ astro.as.utexas.edu \\ ${ }^{3}$ Instituto de Astrofísica de Canarias, C/Via Láctea s/n, E-38205 La Laguna, Spain; agarcia@iac.es \\ ${ }^{4}$ Departamento de Astrofísica, Universidad de La Laguna (ULL), E-38206 La Laguna, Spain \\ 5 Armagh Observatory, College Hill, Armagh BT61 9DG, UK \\ ${ }^{6}$ Physics Department, University of Nebraska at Omaha, NE 68182-0266, USA \\ Received 2012 September 14; accepted 2012 October 15; published 2012 October 26
}

\begin{abstract}
The remarkable hot R Coronae Borealis (RCB) star DY Cen is revealed to be the first and only binary system to be found among the RCB stars and their likely relatives, including the extreme helium stars and the hydrogendeficient carbon stars. Radial velocity determinations from 1982 to 2010 have shown that DY Cen is a single-lined spectroscopic binary in an eccentric orbit with a period of 39.67 days. It is also one of the hottest and most H-rich member of the class of RCB stars. The system may have evolved from a common envelope to its current form.
\end{abstract}

Key words: binaries: general - stars: chemically peculiar - stars: evolution - stars: individual: DY Cen - stars: variables: general - white dwarfs

Online-only material: color figures

\section{INTRODUCTION}

DY Centauri may be a remarkable member of the remarkable class of R Coronae Borealis (RCB) stars. RCB stars are a rare class of peculiar variable stars with two principal and defining characteristics: (1) RCBs exhibit a propensity to fade at unpredictable times by several magnitudes as a result of obscuration by clouds of dust, and (2) RCBs have a supergiantlike atmosphere that is very $\mathrm{H}$-deficient, He-rich, and $\mathrm{C}$-rich. The subject of this Letter, the remarkable star DY Cen, is unusual on several accounts, even as a peculiar RCB. DY Cen is one of the hottest RCBs and it is also one of the most hydrogen-rich RCBs. DY Cen is one of only two RCBs to show features of $\mathrm{C}_{60}$ (or proto-fullerenes) in their spectrum, both being moderately deficient in hydrogen unlike the rest of the class (García-Hernández et al. 2011, 2012). In terms of its chemical composition, DY Cen may have a composition that sets it apart from most RCB and extreme helium (EHe) stars (see Jeffery \& Heber's 1993 abundance analysis). DY Cen is not only uncharacteristically $\mathrm{H}$-rich but is Fe-poor with a very high S/Fe ratio. However, Jeffery et al. (2011) suggest that the Fe abundance was greatly underestimated in 1993 .

The origins of the hydrogen-deficient stars are still a mystery. Two proposals are commonly advocated. In the first proposal, a final helium shell flash occurs on a cooling white dwarf star or a very late thermal pulse is experienced by a postAGB star (Iben et al. 1983; Herwig 2000). These events swell the envelope to supergiant dimensions for a few thousand years and the remaining hydrogen is convected inward and consumed while helium and carbon are convected outward to the surface. Classical examples are believed to be Sakurai's object (V4334 Sgr) and FG Sge (Asplund et al. 1997; Jeffery \& Schönberner 2006). The second proposal involves the merger of two white dwarfs: a carbon-oxygen white dwarf accretes a helium white dwarf as a close binary orbit shrinks due to energy loss by gravitational radiation (Webbink 1984; Iben \& Tutukov 1986). The merger leads to a swollen envelope around the $\mathrm{C}-\mathrm{O}$ white dwarf which lasts a few thousand years. Evidence from the chemical compositions of RCB and EHe stars suggests that most are products of a merger (e.g., García-Hernández et al. 2009, 2010; Pandey \& Lambert 2011; Jeffery et al. 2011). The RCB (or EHe) star from both proposals will be a single star although a distant companion serving as a spectator is not ruled out. The possibility of a distant companion in support of the merger hypothesis seems improbable on the grounds that triple stars are rare.

A search for radial velocity variations of RCB or EHe stars attributable to orbital motion must face the complication that these stars may exhibit atmospheric pulsations. The first systematic radial velocity surveys of hot hydrogen-deficient stars (cf. Jeffery et al. 1987) had revealed evidence of no binaries among the EHe stars. DY Cen was not among the sample studied. However, individual stars showed pulsation-related radial motion among both RCB stars (Lawson \& Cottrell 1997) and EHe stars (Jeffery \& Heber 1992; Jeffery et al. 2001). DY Cen also showed a variable radial velocity and was suggested to be a possible binary (Giridhar et al. 1996). De Marco et al. (2002, p. 3393) remarked with a striking emphasis that "If the fact [... that DY Cen could be understood as a spectroscopic binary...] could be corroborated, it would be the first confirmation of binarity in a RCB star." The current Letter which presents radial velocity measurements spread over two decades suggests that DY Cen may in fact be a spectroscopic binary.

\section{SPECTROSCOPIC OBSERVATIONS}

The general properties of DY Cen's spectrum have been described recently by De Marco et al. (2002). Optical spectra are presently a combination of photospheric absorption lines (e.g., O II, N II, and Si III lines), emission lines due to a stellar wind (mainly $\mathrm{C}_{\mathrm{II}}, \mathrm{He}$ I often superposed on underlying absorption lines, and nebular emission lines of [S II], [N II], [O I], [Fe II], etc. In addition, circumstellar and interstellar lines from Ca II, Na I, $\mathrm{K}$, and other species as well as the enigmatic diffuse interstellar bands (DIBs) are present in absorption (García-Hernández et al. 2012).

Our first spectra of DY Cen were obtained in 1989 and 1992 with the Cassegrain Échelle spectrometer at CTIO's Blanco $4 \mathrm{~m}$ 
Table 1

Radial Velocity Measurements of DY Cen

\begin{tabular}{|c|c|c|c|c|c|c|c|}
\hline $\begin{array}{l}\text { Date } \\
\text { (UT) }\end{array}$ & $\begin{array}{l}\text { Julian Date } \\
(2,400,000+)\end{array}$ & Phase & $\begin{array}{l}\text { Radial Velocity } \\
\left(\mathrm{km} \mathrm{s}^{-1}\right)\end{array}$ & $\begin{array}{l}\text { No. } \\
\text { Lines }\end{array}$ & $\begin{array}{c}\text { Emission } \\
\text { Lines }\left(\mathrm{km} \mathrm{s}^{-1}\right)\end{array}$ & $\begin{array}{c}\text { Nebular } \\
\text { Lines }\left(\mathrm{km} \mathrm{s}^{-1}\right)\end{array}$ & Source \\
\hline 1982 Apr 9 & 45068.734 & 0.102 & $35 \pm 3$ & & & & Herbig \\
\hline 1982 Apr 11 & 45070.624 & 0.150 & $25 \pm 3$ & & & & Herbig \\
\hline 1982 Apr 11 & 45070.664 & 0.151 & $25 \pm 3$ & & & & Herbig \\
\hline 1982 Apr 12 & 45071.694 & 0.177 & $32 \pm 3$ & & & & Herbig \\
\hline 1988 Mar 13 & 47233.774 & 0.682 & $15.1 \pm 2.5$ & & & & Pollacco \& Hill \\
\hline 1989 Jul 16 & 47723.70 & 0.032 & $41 \pm 4$ & & $-2.4 \pm 4.0$ & $22.8 \pm 1.9$ & Rao et al. \\
\hline 1992 May 20 & 48762.608 & 0.223 & $29 \pm 4$ & & $26 \pm 6$ & $21.5 \pm 2.4$ & Giridhar et al. \\
\hline 1999 Feb 5 & 51215.248 & 0.052 & $37.3 \pm 2.4$ & 21 & $23.3 \pm 1.1$ & $23.7 \pm 1.1$ & AAT/UCLES \\
\hline $2001 \mathrm{Apr} 4$ & 52003.998 & 0.936 & $31.7 \pm 2.7$ & & $15.0 \pm 3.2$ & $21.7 \pm 2.1$ & AAT/RGO \\
\hline 2001 Apr 5 & 52005.137 & 0.964 & $32.5 \pm 2.6$ & & $17.4 \pm 2.5$ & $19.6 \pm 2.3$ & AAT/RGO \\
\hline 2002 Jun 26 & 52449.926 & 0.178 & $23.8 \pm 2.0$ & 19 & $20.1 \pm 1.5$ & $20.1 \pm$ & AAT/UCLES \\
\hline 2003 Jul 18 & 52838.903 & 0.984 & $40.0 \pm 1.5$ & 11 & $14.0 \pm 5.0$ & $22.3 \pm 1.2$ & AAT/UCLES \\
\hline 2010 Feb 27 & 55254.772 & 0.886 & $24.7 \pm 2.6$ & 36 & $14.8 \pm 2.6$ & $21.3 \pm 1.3$ & VLT/UVES \\
\hline 2010 Mar 2 & 55257.749 & 0.961 & $25.4 \pm 2.5$ & 47 & $15.7 \pm 3.1$ & $20.9 \pm 1.1$ & VLT/UVES \\
\hline 2010 Mar 5 & 55260.795 & 0.038 & $36.9 \pm 4.6$ & 34 & $24.7 \pm 2.7$ & $20.9 \pm 1.3$ & VLT/UVES \\
\hline 2010 Mar 25 & 55280.601 & 0.537 & $14.2 \pm 3.6$ & 28 & $22.8 \pm 3.3$ & $20.6 \pm 1.1$ & VLT/UVES \\
\hline
\end{tabular}

reflector. The resolving power is close to 18,000 for the 1989 spectra and about 35,000 for the 1992 spectrum (Rao et al. 1993; Giridhar et al. 1996).

Observations over four years were obtained at the $3.9 \mathrm{~m}$ Anglo-Australian Telescope (AAT) with either the University College London Échelle Spectrometer (UCLES) or the Royal Greenwich Observatory (RGO) spectrograph. The resolving power of the UCLES spectra is about 40,000 and 13,000 for the RGO spectra. The spectral bandpass of the UCLES spectra ran from $4350 \AA$ to $7335 \AA$ in $1999,3800 \AA$ to $5100 \AA$ in 2002 , and $4780 \AA$ to $8800 \AA$ in 2003 . The RGO spectra spanned the interval 3900-4790 $\AA$. These spectra were retrieved from the observatory's archive and rereduced.

Spectra of DY Cen were obtained on four nights in 2010 February-March with the cross-dispersed Échelle spectrograph UVES with the Very Large Telescope (VLT) at ESO's Paranal observatory. The spectral resolving power as estimated from telluric lines at $6970 \AA$ is 37,000 . These spectra cover the regions 3300-4500 $\AA, 5700-7525 \AA$, and 7660-9460 $\mathrm{A}$. The stellar radial velocity was measured from those absorption lines longward of $4200 \AA$ which appeared least affected by emission and asymmetries. The majority of the lines are from $\mathrm{O}$ II multiplets and higher ionization species including C III, C IV, and $\mathrm{Al}$ III. The centers of the lines have been measured using a cursor. Our measurements are summarized in Table 1, which also includes radial velocities by Pollacco \& Hill (1991) and George Herbig who obtained spectra in 1982 using CTIO's 4 m Blanco telescope and a Cassegrain spectrograph. It is expected that the emission line strengths relative to the continuum in 1982 would have been much weaker.

In combining velocity measurements from different telescopes and spectrographs, it is important to check for systematic effects. A check in the case of DY Cen is offered by the nebular forbidden lines. These originate from a low-density region (Giridhar et al. 1996), which is presumably remote from the star and unaffected by its atmospheric changes. Measurements of these lines are shown in Table 1, which illustrates that any systematic effects that exist are within $\pm 1.5 \mathrm{~km} \mathrm{~s}^{-1}$. Moreover, the mean of these 11 measurements is $21.6 \mathrm{~km} \mathrm{~s}^{-1}$, a value consistent with the $\gamma$-velocity of $21.3 \mathrm{~km} \mathrm{~s}^{-1}$ from the fitted orbit (Table 2).
Table 2

Orbital Parameters of DY Cen

\begin{tabular}{lc}
\hline \hline Parameter & Value \\
\hline$T_{\mathrm{o}}(\mathrm{JD})$ & $2445104.3364 \pm 1.715$ \\
$P$ & $39.66779 \pm 0.0088$ days \\
$\gamma$ & $21.30 \pm 0.45 \mathrm{~km} \mathrm{~s}^{-1}$ \\
$K$ & $13.26 \pm 1.18 \mathrm{~km} \mathrm{~s}^{-1}$ \\
$e$ & $0.44 \pm 0.10$ \\
$\omega$ & $344.5 \pm 16.7$ \\
$f(M)$ & $6.92_{-0.91}^{+0.43} 10^{-3} M_{\odot}$ \\
\hline
\end{tabular}

In contrast, the stellar emission lines do vary in velocity. The variation of emission line velocity seems to be uncorrelated with absorption line velocities. Moreover, the emission is mostly confined to lower multiplets of $\mathrm{CII}$ and $\mathrm{He}$ I lines whereas the measured absorption lines are from higher ionization and excitation species.

DY Cen was identified as a variable of the RCB type by Hoffleit (1930) from well-determined minima in 1897, 1901, 1924, and 1929. No RCB-type minima were recorded since 1958 (Bateson 1978; A. A. Henden, 2010, private communication). In this respect, DY Cen is no longer an active RCB star and all the spectra discussed here were taken when the star was at maximum light.

\section{IS DY Cen A SPECTROSCOPIC BINARY?}

For a clear demonstration that DY Cen is a spectroscopic binary, one should show that the velocity changes do not arise from atmospheric disturbances (e.g., radial and nonradial pulsations or spots). Inspection of the cache of spectra shows that many emission lines and also those absorption lines with emission components are variable. While this variability may add "noise" to the orbital solution, it could provide supporting evidence for the binary hypothesis if the spectroscopic variations may be shown to be correlated with orbital phase.

In the following subsections, we present the orbital solution, discuss whether atmospheric pulsations have been mistaken for orbital velocity changes, and offer comments on line profile variations tied to orbital phase. 


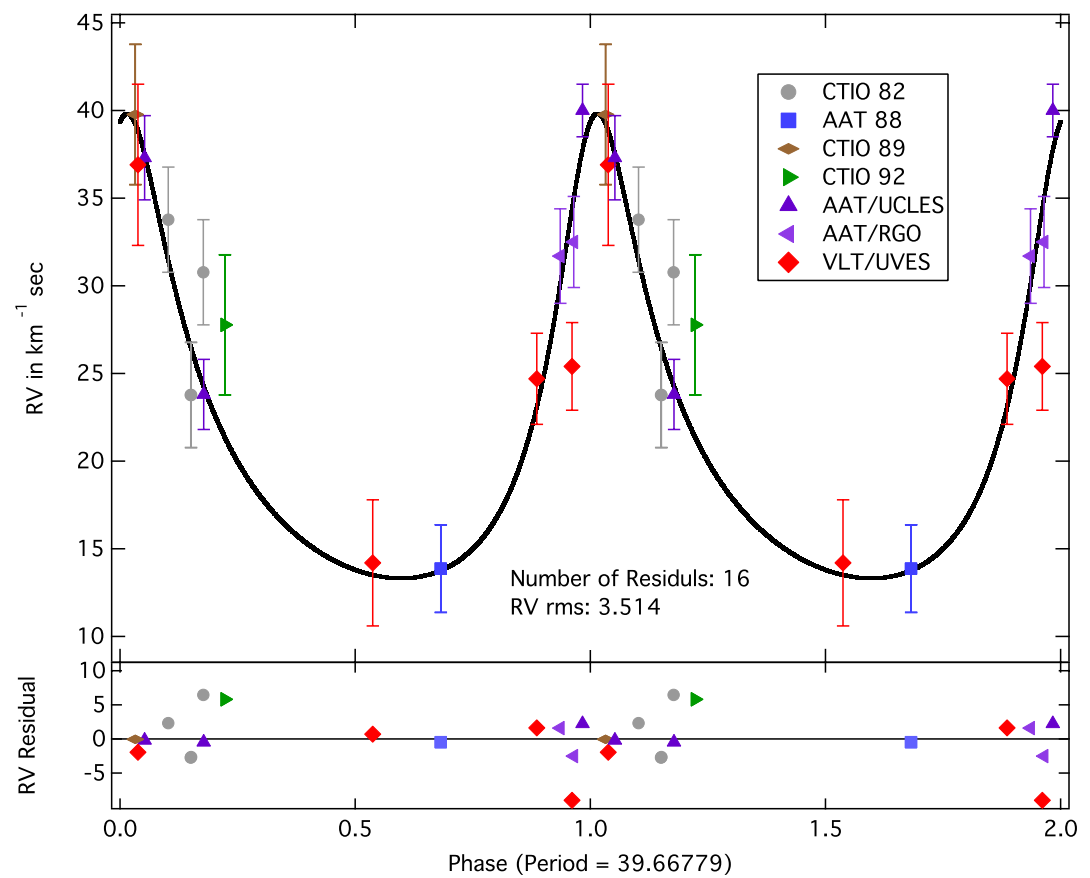

Figure 1. Stellar radial velocity curve similar to Figure 3. The deviations of observations to the computed fit for the parameters in Table 4 are shown in the bottom. The notation of the observations is shown in the inset.

(A color version of this figure is available in the online journal.)

\subsection{The Spectroscopic Binary Orbit}

Radial velocity determinations in Table 1 were examined for periodic variations. After investigating trial periods between 28 and 50 days, we arrived at a period of about 39.7 days that gave a satisfactory fit to all measurements. The final period and the orbital solution were determined using a GaussFit binary model (as in McArthur et al. 2010). Orbital parameters are summarized in Table 2. The fit to the data is shown in Figure 1 along with the residuals to that fit. The least squares $\chi^{2}$ of the orbital model was 23.5 for 11 degrees of freedom (DOF), indicating that either the errors of the data are generally underestimated, there are significant outliers in the data set, or the model is not complete. We used GaussFit's capacity for robust estimation, which improves the resistance of the parameter values to the effects of "outliers," which are observations that are not known a priori to be bad. This is done within the code by allowing the observations with small residuals to dominate the result, iteratively reweighting the outliers with higher errors. The $\chi^{2}$ of the robust estimation solution is 9.2 for $11 \mathrm{DOF}$, which indicated that the data set did contain outliers, which can be seen in the lower portion of Figure 1. In order to consider the adequacy of the model to fit the observational data, we also used a sinusoidal model and a linear model to fit the data. The least-squares solution of the sinusoidal model produced a $\chi^{2}$ of 114.2 for $12 \mathrm{DOF}$ and the $\chi^{2}$ of a linear model fit of the data is 144.6 for 14 DOF. These $\chi^{2}$ of these two alternative model fits of the data are much higher than the $\chi^{2}$ of 23.5 found for the binary orbit model. The sinusoidal model did not approximate the orbital model because of the significant eccentricity of the orbital fit. With low eccentricities, the sinusoidal model can approximate the fit of an orbital model.

The small mass function shows that the unseen secondary has a low mass. The minimum mass $M_{2} \sin i$ is around $0.2 / \sin i M_{\odot}$ for, say, a primary mass of about $0.8 M_{\odot}$; the secondary mass is insensitive to the assumed mass of the primary. If $\sin ^{3} i$ has its average value $(3 \pi / 16$ for a random distribution of inclinations), the secondary mass increases to about $0.3-0.4 M_{\odot}$. The semimajor axis $a$ is $9.3 / \sin i R_{\odot}$. The secondary may be a low-mass helium white dwarf or a low-mass (stripped?) mainsequence star.

\subsection{Atmospheric Pulsations?}

Changes in absorption line profiles arising from pulsations or surface spots translate to a radial velocity change when velocities are measured by our technique. Fortunately, pulsations, in general, and spots, on all occasions, will result in a change of line profile. Among the available spectra, only the VLT/UVES spectra are of the necessary quality to search for subtle line profile variations.

Our VLT/UVES spectra sample a phase interval of 0.6. In Figure 2, we show the mean spectrum spanning the $\mathrm{C}$ IV lines near $5800 \AA$ and including the DIB at $5796 \AA$ and difference spectra relative to the mean spectrum. The four VLT/UVES spectra span the phase interval between quadratures (radial velocity maximum and minimum). Individual spectra were shifted to the $\mathrm{C}$ IV laboratory wavelengths in order to construct the mean profile and then the difference with the mean spectrum was computed. As Figure 2 shows, these difference profiles around the $\mathrm{C}$ IV lines are featureless indicating that the absorption profiles over the observing interval were invariant. (There is a signal in the difference spectra around the DIB because this interstellar feature is not subject to the stellar velocity variations.)

Atmospheric oscillations introduce changes in the observed line symmetry as a velocity shift and line strength (due to changes in effective temperature and surface gravity). These changes should be easily visible in the differenced spectra, especially for temperature sensitive lines, such as Civ. None can be seen in the VLT/UVES spectra of DY Cen. The absence of evidence for oscillations at the few $\mathrm{km} \mathrm{s}^{-1}$ amplitude in 2010 came as a surprise because the EHe star BD $-9^{\circ} 4395$, 


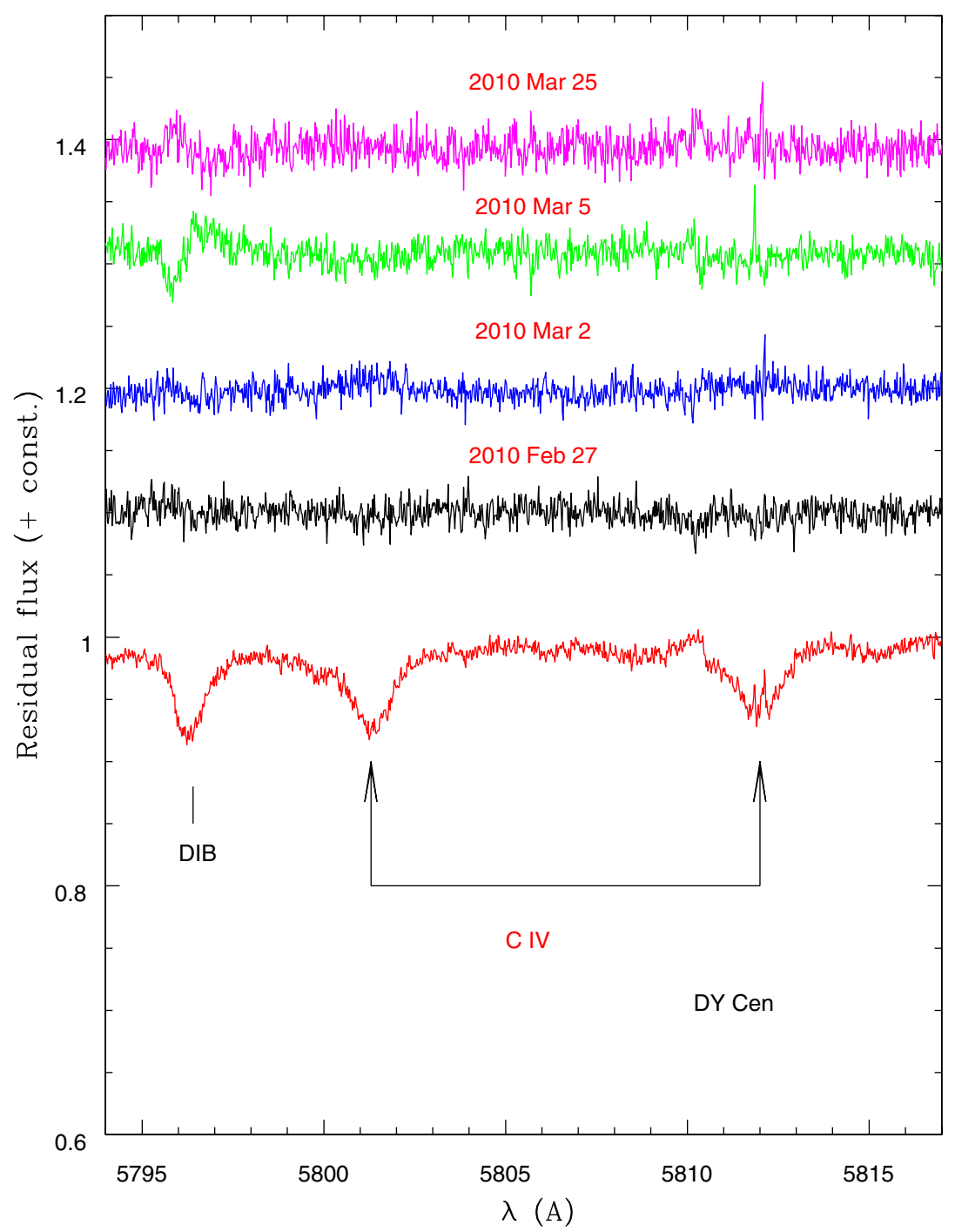

Figure 2. Mean and differenced spectra around the C IV 5800-5810 A lines. The sense of the differenced spectra is "observed-mean." (A color version of this figure is available in the online journal.)

with atmospheric parameters very similar to those of DY Cen, is plagued with such oscillations. BD $-9^{\circ} 4395$ has atmospheric parameters $\left(T_{\text {eff }} \mathrm{K}, \log g \mathrm{cgs}\right)=(24,300,2.65)$ (Pandey \& Lambert 2011). DY Cen was measured to have parameters $(19,500,2.15)$ by Jeffery \& Heber $(1993)$ but has evolved to higher temperatures in recent years, say $(24,800$, 2.5 , unpublished analysis). This is an almost perfect replica of BD $-9^{\circ} 4395$, apart from having approximately 50 times more hydrogen. Pulsation periods of EHe stars have been calculated by Saio (2007). Predicted periods for BD $-9^{\circ} 4395$ are typically between 2 and 3 days, a range which includes its measured period of $3.0 \pm 1.5$ days for BD $-9^{\circ} 4395$ (Jeffery \& Heber 1992). The scale of the line profile variations for BD $-9^{\circ} 4395$ corresponds to a dispersion profile of peak-to-peak amplitude of about \pm 0.05 of the continuum flux in differenced spectra of strong lines like the CIV lines in Figure 2 (see Figure 7 in Jeffery \& Heber 1992). Such features are clearly not present in difference spectra for DY Cen.

The contrast between the presence of nonradial oscillations in BD $-9^{\circ} 4395$ and their absence in DY Cen in 2010 is surprising. Perhaps, their excitation is intermittent or even quenched at the higher $\mathrm{H}$ abundance of DY Cen: the $\mathrm{H}$ abundance is $\log \epsilon(\mathrm{H})=$
10.8 for DY Cen (Jeffery \& Heber 1993) and 9.1 for BD -9 4395 (Pandey \& Lambert 2011). Hydrogen quenching is evident in pulsation models for higher gravity EHe stars, only when the hydrogen mass fraction exceeds $30 \%$ (Jeffery \& Saio 1999); consequently DY Cen should still be unstable to radial pulsations.

Pollacco \& Hill (1991) reported low-amplitude $(\Delta V \sim 0.1)$ short-period (3.8-5.5 days) photometric variations from 1987 observations. Surface pulsations producing such light variations would be associated with velocity variations of up to $20 \mathrm{~km} \mathrm{~s}^{-1}$ (see Jeffery et al. 2001). Since we cannot exclude the presence of such motion from, at least, the earlier observations in our data set, we have to acknowledge that our current orbital solution is uncertain to at least this extent.

The 1987 observations also showed a longer duration $(<25$ days $)$ and larger amplitude $(\Delta V \sim 0.2)$ and appeared to confirm reports from Bateson (1978) from visual observations of a longer period variation. Such a variation may not be intrinsic to the star but may arise from changing extinction as the star moves in its orbit. In this regard, one recalls the behavior of RV Tauri variables and the photometric distinction between the RVa and $\mathrm{RVb}$ types. 


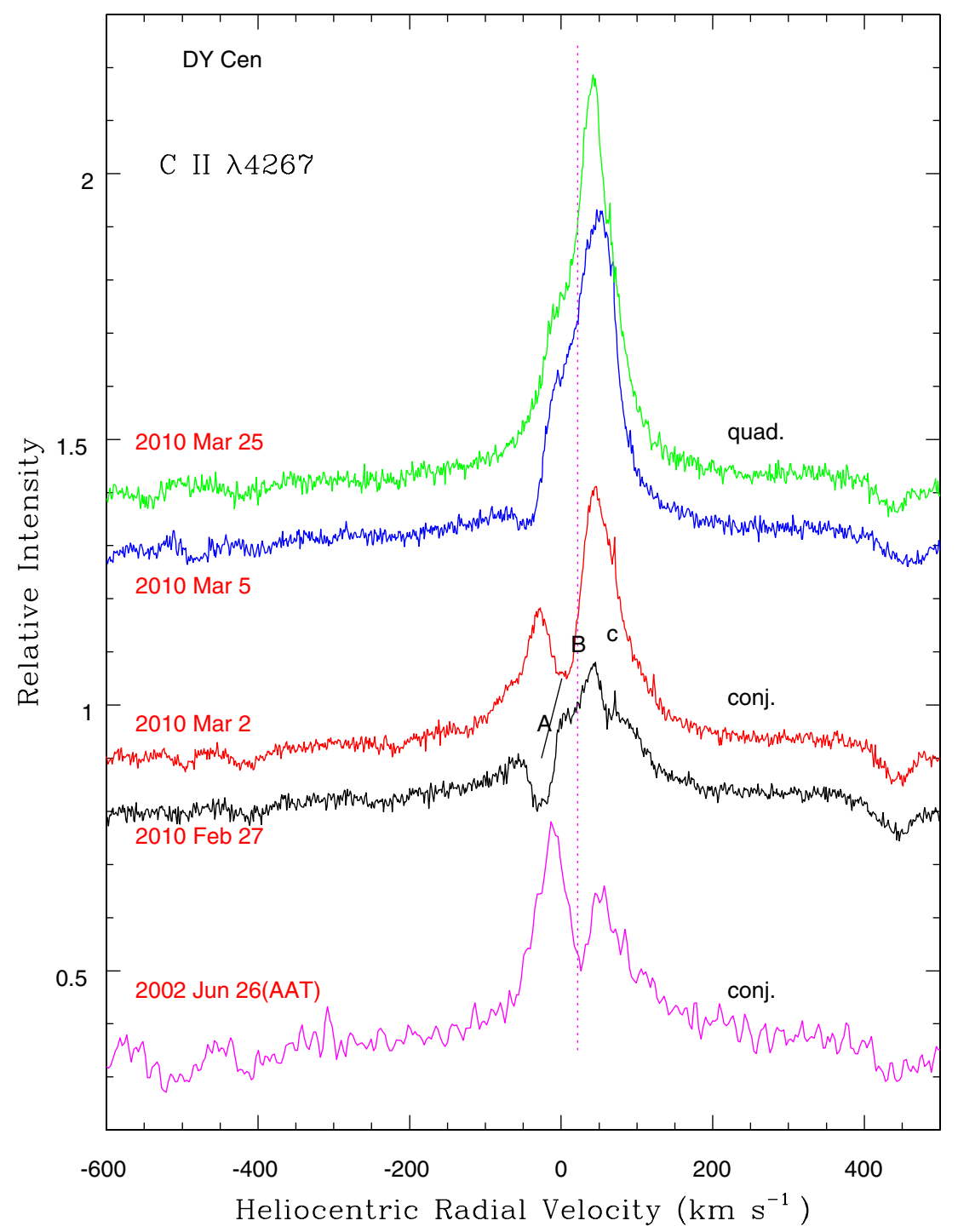

Figure 3. C II $4267 \AA$ A profiles during the 2010 February-March period. The spectra are shifted by arbitrary amounts in $y$-axis for clarity. Absorption components are superposed on the broad emission profile. These components are prominent in the profiles obtained near conjunction. The emission flux is lowest near conjunction. The vertical dashed line represents the systemic velocity (obtained from nebular lines).

(A color version of this figure is available in the online journal.)

\subsection{Phase-dependent Spectroscopic Changes?}

Our optical spectra of DY Cen show that there are short- and long-term changes in the spectral features. There is a tantalizing hint of orbital phase-dependent changes in the $4267 \AA \mathrm{C}_{\text {II }}$ emission profile-see Figure 3. Near conjunction, one or more absorption components appear superimposed on the emission profile. (A similar profile is also seen at the other conjunction (primary on the farside) from the observations obtained in 2002 June 26.) However, the profile of the $C_{\text {II }} 7231 \AA$ emission line is essentially the same on all four spectra with no hint of superposed absorption, and the $\mathrm{C}_{\text {II }} 6578 \AA$ line shows a $\mathrm{P}$ Cygni profile at all four phases indicative of strong mass loss. Additional observations with complete phase coverage will be needed to investigate phase-dependent spectral variations.

\section{CONCLUDING REMARKS}

The variable radial velocities of DY Cen suggest that it is a single-lined spectroscopic binary. The absorption line profiles over the interval spanned by the VLT/UVES observations indicate that nonradial oscillations are unlikely to account for the amplitude of the radial velocity variation in 2010. Thus, we assume that DY Cen is a product of binary star evolution, unlike all other members of RCB class.

Consideration of the size of the orbit and the radius of DY Cen shows that the components of this binary have experienced and are likely to continue to experience mass loss and mass transfer, which is revealed by the presence of emission lines throughout the spectrum. For example, the semimajor axis $a \sin i=9.3 R_{\odot}$ may be compared with the radius of DY Cen. Assuming a mass of $0.8 M_{\odot}$ and using the recent measurement $\log g=2.5$ gives a stellar radius $\approx 8 R_{\odot}$. Looking back 20 years and using $\log g=2.15$ (Jeffery \& Heber 1993) gives a radius $\approx 12 R_{\odot}$. Considering the eccentricity $e=0.44$ given by our orbital solution, periastron passage will occur with a separation of some $10 / \sin i R_{\odot}$. This close proximity and evidence that DY Cen is contracting at a substantial rate may well explain both the very active emission-line activity and the peculiar nature of DY Cen in the recent past. If the surface of DY Cen had been as cool as $5000 \mathrm{~K}$, its radius would have exceeded $100 R_{\odot}$, several 
times the present orbital separation. Indeed, it is possible that DY Cen was a true common-envelope system, with the secondary embedded within the envelope of the primary, within the last 100 years. Even this episode was almost certainly preceded by earlier mass transfer and orbital changes; the radius of a normal star on the first giant branch is greater than the inferred semimajor axis-unless the angle of inclination is very high.

As a close binary with a substantial surface abundance of hydrogen, and with an apparent contraction rate in excess of any observed among the EHe and classical RCB stars, the latter are probably not useful types for considering the evolution of DY Cen. Similarly, the more massive hydrogen-deficient binaries including $v$ Sgr and KS Per are extremely hydrogendeficient and nitrogen-rich and unlikely counterparts. If DY Cen continues to contract at the rate suggested by the measurements of $T_{\text {eff }}$ and $\log g$ (Jeffery \& Heber 1993 and the present), it is possible that there is no known star in the Galaxy quite like it.

Regarding its future evolution, one can do little more than speculate. Although at present DY Cen is probably transferring mass to its companion, its rapid contraction implies that mass transfer will soon cease. That DY Cen will first become a hot subdwarf and subsequently a white dwarf appears to be clear. Less clear is whether it presently has a helium or a carbon-oxygen core. The first case should lead to a stable helium-core burning phase, where it might be identified among the intermediate helium subdwarf stars (cf. CPD-20 1123 ; Naslim et al. 2012). Since atomic diffusion transforms the surface composition of subdwarf B stars, it is difficult to identify possible successors reliably; intermediate helium subdwarfs come with a range of surface carbon and nitrogen abundances, for example $(0.1<\mathrm{C} / \mathrm{N}<10$; Naslim et al. 2012). It is not known whether intermediate helium stars become classical sdB stars-which are very helium deficient. Since the processes that form sdB stars in binaries remove most of the hydrogen envelope, one might expect their surfaces to be somewhat hydrogen-deficient until radiatively driven diffusion forces the helium to sink below sight, so DY Cen could be an immediate post-common-envelope binary in transition to the subdwarf $\mathrm{B}$ phase. The majority of $\mathrm{sdB}$ stars are binaries, and several have periods in the 10-100 day range (Maxted et al. 2001; Copperwheat et al. 2011; Barlow et al. 2012). On the other hand, if it is slightly more massive and already has a carbon-oxygen core, then it will evolve directly through the helium-rich subdwarf $\mathrm{O}$ star and on to become a hot white dwarf. Its subsequent appearance and evolution will depend on the nature of the unseen secondary.
We acknowledge the anonymous referee for suggestions that helped to improve the paper. This work is based on observations obtained with the ESO programme 284.D- 5048(A). D.A.G.H. acknowledges support provided by the Spanish Ministry of Economy and Competitiveness under grant AYA-2011-27754. Our sincere thanks are due to George Herbig for supplying his radial velocity measurements. We acknowledge with thanks use of SIMBAD and AAVSO databases.

Facility: VLT:Kueyen

\section{REFERENCES}

Asplund, M., Gustafsson, B., Lambert, D. L., \& Kameswara Rao, N. 1997, A\&A, 321, 17

Barlow, B. N., Wade, R. A., Liss, S. E., Østensen, R. H., \& Van Winckel, H. 2012, ApJ, 758, 58

Bateson, F. M. 1978, Publs. Var. Star, Sect., R. Astron. Soc. New Zealand, 6, 39

Copperwheat, C. M., Morales-Rueda, L., Marsh, T. R., Maxted, P. F. L., \& Heber, U. 2011, MNRAS, 415, 1381

De Marco, O., Clayton, G. C., Herwig, F., et al. 2002, AJ, 123, 3387

García-Hernández, D. A., Hinkle, K. H., Lambert, D. L., \& Eriksson, K. 2009, ApJ, 696, 1733

García-Hernández, D. A., Kameswara Rao, N., \& Lambert, D. L. 2011, ApJ, 729,126

García-Hernández, D. A., Kameswara, R. N., \& Lambert, D. L. 2012, ApJ, 759, L21

García-Hernández, D. A., Lambert, D. L., Kameswara Rao, N., Hinkle, K. H., \& Eriksson, K. 2010, ApJ, 714, 144

Giridhar, S., Kameswara Rao, N., \& Lambert, D. L. 1996, J. Astrophys. Astron., 17,23

Herwig, F. 2000, A\&A, 360, 952

Hoffleit, D. 1930, Harvard Bull., 874, 1

Iben, I., Jr., Kaler, J. B., Truran, J. W., \& Renzini, A. 1983, ApJ, 264, 605

Iben, I., Jr., \& Tutukov, A. V. 1986, ApJ, 311, 753

Jeffery, C. S., Drilling, J. S., \& Heber, U. 1987, MNRAS, 226, 317

Jeffery, C. S., \& Heber, U. 1992, A\&A, 260, 133

Jeffery, C. S., \& Heber, U. 1993, A\&A, 270, 167

Jeffery, C. S., Karakas, A. I., \& Saio, H. 2011, MNRAS, 414, 3599

Jeffery, C. S., \& Saio, H. 1999, MNRAS, 308, 221

Jeffery, C. S., \& Schönberner, D. 2006, A\&A, 459, 885

Jeffery, C. S., Starling, R. L. C., Hill, P. W., \& Pollacco, D. 2001, MNRAS, 321,111

Lawson, W. A., \& Cottrell, P. L. 1997, MNRAS, 285, 266

Maxted, P., Marsh, T. R., \& North, R. C. 2001, in ASP Conf. Ser. 226, 12th European Workshop on White Dwarfs, ed. J. L. Provencal, H. L. Shipman, J. MacDonald, \& S. Goodchild (San Francisco, CA: ASP), 187

McArthur, B. E., Benedict, G. F., Barnes, R., et al. 2010, ApJ, 715, 1203

Naslim, N., Geier, S., Jeffery, C. S., et al. 2012, MNRAS, 423, 3031

Pandey, G., \& Lambert, D. L. 2011, ApJ, 727, 122

Pollacco, D. L., \& Hill, P. W. 1991, MNRAS, 248, 572

Rao, N. K., Giridhar, S., \& Lambert, D. L. 1993, A\&A, 280, 201

Saio, H. 2007, in ASP Conf. Ser. 391, Hydrogen Deficient Stars, ed. K. Werner \& T. Rauch (San Francisco, CA: ASP), 69

Webbink, R. F. 1984, ApJ, 277, 355 